\title{
Safety of Rituximab in Combination with Other Biologic Disease-modifying Antirheumatic Drugs in Rheumatoid Arthritis: An Open-label Study
}

\author{
William F.C. Rigby, Philip J. Mease, Ewa Olech, Mark Ashby, and Swati Tole
}

ABSTRACT. Objective. To characterize the safety of rituximab (RTX) in combination with biologic disease-modifying antirheumatic drugs (DMARD) in patients with rheumatoid arthritis (RA).

Methods. We did an open-label study of the safety and efficacy of RTX in adult patients with active RA and an inadequate response to $\geq 1$ biologic for $\geq 12$ weeks (stable dose $\geq 4$ weeks). RTX ( $2 \times$ $500 \mathrm{mg}$ ) was added to patients' current biologic and nonbiologic DMARD treatment. After 24 weeks, patients with 28-joint Disease Activity Score $\geq 2.6$ were eligible for RTX retreatment. The primary endpoint was the proportion of patients developing a serious adverse event (SAE) within 24 weeks of initiating RTX.

Results. Patients $(\mathrm{n}=176)$ received RTX with 18 different biologic/DMARD combinations. Adalimumab alone $(n=46 ; 26.1 \%)$ or etanercept alone $(n=37 ; 21.0 \%)$ plus RTX were the most common combinations. Overall, $90.9 \%$ and $76.1 \%$ of patients completed 24 and 48 weeks, respectively; 147 patients $(83.5 \%)$ received a second course of RTX. Over 24 weeks, $9.1 \%$ of patients reported SAE (24.3 events/100 patient-yrs, 95\% CI 15.5-38.1). The SAE rate was similar over 48 weeks (22.4 events/100 patient-yrs, 95\% CI 15.9-31.5). Four serious infections were reported over 48 weeks (2.7 events/100 patient-yrs, 95\% CI 1.0-7.2). No SAE occurred within 24 h of any RTX infusion. Efficacy responses improved numerically at Week 48 compared with Week 24.

Conclusion. The overall safety profile of RTX in combination with 1 other biologic was consistent with that previously reported for RTX plus methotrexate or other nonbiologic DMARD. (Clinicaltrials.gov NCT00443651) (First Release April 1 2013; J Rheumatol 2013;40:599-604; doi:10.3899/jrheum.120924)

Key Indexing Terms:

B LYMPHOCYTES

BIOLOGICAL THERAPY

RHEUMATOID ARTHRITIS

Biologic disease-modifying antirheumatic drugs (DMARD) provide effective relief of the major clinical signs and symptoms of rheumatoid arthritis (RA) and also slow the rate of joint damage ${ }^{1}$. However, about $20 \%-40 \%$ of patients do not achieve an adequate clinical response to biologic DMARD treatment ${ }^{2,3,4,5,6,7}$. Such patients may be candidates for additional therapy.

Previous randomized clinical trials in patients with RA

From the Department of Medicine, Dartmouth Medical School, Lebanon, New Hampshire; Swedish Medical Center, University of Washington, Seattle, Washington; University of Nevada School of Medicine, Las Vegas, Nevada; and Genentech Inc., South San Francisco, California, USA. Sponsored by Genentech Inc. and Biogen-Idec; and by F. Hoffmann-La Roche Ltd.

W.F.C. Rigby, MD, Professor of Medicine, Dartmouth Medical School; P.J. Mease, MD, Director, Rheumatology Research, Swedish Medical Center and Clinical Professor, University of Washington School of Medicine; E. Olech, MD, Associate Professor of Medicine, University of Nevada School of Medicine; M. Ashby, PhD, Principal Statistical Scientist/Biostatistics; S. Tole, MD, MS, Medical Director, Genentech Inc. Address correspondence to Dr. W.F.C. Rigby, Dartmouth Medical School, 1 Medical Center Drive, HB 7510, Lebanon, NH 03756-0001, USA.

E-mail: william.rigby@dartmouth.edu

Full Release Article. For details see Reprints/Permissions at jrheum.org Accepted for publication January 25, 2013. have shown that certain biologic therapy combinations may result in an increased incidence of important adverse events (AE). The addition of anakinra $100 \mathrm{mg} /$ day to etanercept 25 $\mathrm{mg}$ once or twice weekly in patients with active RA despite methotrexate (MTX) therapy was associated with a higher incidence $(7.4 \%$ vs $0 \%)$ of serious infectious events (SIE) at 24 weeks $^{8}$. Similarly, the addition of abatacept to other biologics increased the incidence of SIE over 12 months (5.8\% vs $2.6 \%)$ when compared with treatment with abatacept alone ${ }^{9}$. It is important to note that in both studies, patients continued background MTX or other nonbiologic DMARD therapy while being treated with the biologic combination, and many were also receiving concomitant corticosteroids.

Rituximab (RTX) is an effective therapy in patients with RA and an inadequate response to either nonbiologic DMARD or tumor necrosis factor (TNF) inhibitors ${ }^{10,11,12,13,14}$. The safety of RTX in combination with other biologic therapies is an important clinical issue as patients with an inadequate response to RTX may receive subsequent biologic therapies while remaining B cell-depleted, and a subset of refractory patients may receive concurrent treatment with $>1$ biologic DMARD. Results of a recent placebo-controlled study

Personal non-commercial use only. The Journal of Rheumatology Copyright $@$ $~ 2013$. All rights reserved. 
evaluating RTX in combination with MTX plus a TNF inhibitor (etanercept or adalimumab) indicated that the safety profile for the RTX combination was consistent with that observed in previous RTX clinical trials ${ }^{15}$. The current study (SUNDIAL II) was conducted to further define the safety of RTX in combination with any other biologic agents approved by the US Food and Drug Administration (FDA) in the treatment of RA. We report that combination therapy with RTX plus 1 other biologic DMARD in patients with active RA and an inadequate response to biologic DMARD demonstrated similar rates of serious AE, including SIE, to those previously reported in other studies of RTX in RA.

\section{MATERIALS AND METHODS}

Patients. Men and women were eligible for inclusion in the study if they were between 18 and 80 years of age and had presented with active RA at least 6 months prior to the start of the study. Active disease was defined as a swollen and tender joint count $\geq 6$. Study entry did not require an elevated erythrocyte sedimentation rate (ESR) or C-reactive protein level. Patients were also required to have shown an inadequate response to at least 1 prior biologic DMARD for at least 12 weeks, with stable dose for at least 4 weeks prior to baseline. Patients were required to tolerate their currently prescribed biologic DMARD at the following doses: etanercept 50 $\mathrm{mg} /$ week; adalimumab $40 \mathrm{mg}$ every other week; infliximab $(3-5 \mathrm{mg} / \mathrm{kg}$ ) every 8 weeks in combination with MTX; and abatacept 500-1000 mg every 4 weeks. Additional permitted medication included nonbiologic DMARD at a stable dose for at least 4 weeks prior to baseline, stable doses of corticosteroids ( $\leq 10 \mathrm{mg} /$ day prednisone or equivalent), and nonsteroidal antiinflammatory drugs.

Patients were excluded from the study if they had a history of other autoimmune or inflammatory diseases; evidence of other significant uncontrolled concomitant disease; or were receiving concurrent treatment with infliximab monotherapy, leflunomide plus MTX, azathioprine, or $>1$ biologic agent.

The study was performed in accord with the Declaration of Helsinki. All participating study centers received approval from their institutional review board or ethical committee. All patients provided written informed consent (study registered with Clinicaltrials.gov; NCT00443651).

Study design and treatment. This was a 48-week, open-label study in which patients received RTX $2 \times 500 \mathrm{mg}$ [given as intravenous (IV) infusions 2 weeks apart] plus their current biologic and nonbiologic DMARD therapy at the stable dose. Premedication with IV methylprednisolone $100 \mathrm{mg}$ was given prior to each RTX infusion. There was no washout period for concurrent biologic or nonbiologic DMARD prior to dosing of RTX. Between Weeks 24 and 40, patients with Disease Activity Score in 28 joints (DAS28) $\geq 2.6$ were eligible for an optional second course of RTX.

Assessments. AE, serious AE (SAE), infections, and SIE were monitored and recorded throughout the 48 weeks. AE that occurred within 24 hours of RTX infusion were classified as infusion-related reactions (IRR). All AE were graded according to the National Cancer Institute's Common Terminology Criteria for Adverse Events, version 3. SIE were predefined as infections that required IV antibiotics or met the regulatory criteria for an SAE, where at least 1 of the following applied to the infection: required inpatient hospitalization or prolonged an existing hospitalization; immediately life-threatening; resulted in persistent or significant disability or incapacity; medically significant, where an intervention was required to prevent one of the above-mentioned outcomes; or fatal. Routine laboratory tests were performed, as well as assays for human antichimeric antibodies (HACA) against RTX.

Endpoints and clinical outcomes. The primary endpoint of the study was the proportion of patients developing an SAE within 24 weeks after 1 course of RTX. Other safety assessments included incidence of IRR for each infusion of RTX, SAE that occurred within 24 weeks of a second course of RTX, and SIE. Open-label efficacy was assessed at Weeks 24 and 48. The efficacy endpoints included the proportion of patients who achieved American College of Rheumatology (ACR) ACR20, ACR50, and ACR70 responses; and the proportions of patients who achieved DAS28-ESR remission and low disease activity (LDA); and European League Against Rheumatism (EULAR) responses. The level of physical functioning and disability was assessed by the change from baseline in Health Assessment Questionnaire-Disability Index (HAQ-DI) scores.

Statistical analysis. Analysis of safety and efficacy outcomes was based on enrolled patients treated with RTX. Baseline demographics, disease characteristics, and DMARD use were summarized. AE over 48 weeks were coded and summarized according to MedDRA (version 14.0) system organ class and preferred terms. Safety outcomes other than infusion reactions were reported as incidence rates per 100 patient-years and percentage of patients affected. Infusion reactions were reported as percentage of patients affected within $24 \mathrm{~h}$ of infusion. The proportion of patients with ACR 20/50/70 responses, EULAR response, DAS28-ESR remission, and DAS28-ESR LDA were summarized at Weeks 24 and 48.

The study was designed to include 160 patients receiving RTX in combination with an FDA-approved biologic DMARD. Assuming a 10\% dropout rate, 160 patients gave about 70 patient-years of 24-week followup, permitting detection of $\mathrm{AE}$ occurring at a rate of $\geq 4.3$ events per 100 patient-years.

\section{RESULTS}

Patients had a mean age of 54 years and were mostly white women (Table 1). The majority of patients $(60 \%)$ was seropositive for rheumatoid factor (RF) and/or anticitrullinated peptide antibody. Mean RA disease duration was about 11 years and patients had been previously treated with

Table 1. Demographics and baseline disease characteristics. Data are mean (SD) (range) unless otherwise indicated.

\begin{tabular}{lc}
\hline Characteristic & $\begin{array}{c}\text { Safety Population, } \\
\mathrm{n}=176\end{array}$ \\
\hline Age, yrs & $53.6(11.7)(18-80)$ \\
Female, $\%$ & 87.5 \\
White, $\%$ & 79.0 \\
No. prior DMARD* & $4.0(1.6)(1-10)$ \\
No. prior TNF inhibitors & $1.5(0.6)(0-3)$ \\
Disease duration, yrs & $10.6(9.0)(0-41.7)$ \\
RF-positive, $\%$ & 61.4 \\
ACPA-positive, $\%$ & 62.5 \\
CRP, mg/dl & $1.26(2.68)(0.02-28.40)$ \\
ESR, mm/h & $32.7(24.3)(1.0-30.0)$ \\
DAS28-ESR & $6.21(1.05)(2.5-8.8)$ \\
SJC (of 66 joints) & $19.0(11.0)(5-59)$ \\
TJC (of 68 joints) & $29.5(15.7)(6-68)$ \\
HAQ-DI & $1.47(0.64)(0.0-3.0)$ \\
Oral steroid use, $\%$ & 39.2 \\
One steroid use, mg/day & $6.5(2.7)(1.0-10.0)$ \\
\hline
\end{tabular}

\footnotetext{
* Biologic and nonbiologic DMARD, including methotrexate. Excluding methotrexate, patients had received a mean of 3.0 prior biologic and nonbiologic DMARD. ${ }^{\dagger}$ While taking baseline biologic/nonbiologic DMARD. ACPA: anticitrullinated protein antibody; CRP: C-reactive protein; DAS28: Disease Activity Score in 28 joints; DMARD: disease-modifying antirheumatic drug; ESR: erythrocyte sedimentation rate; HAQ-DI: Health Assessment Questionnaire-Disability Index; RF: rheumatoid factor; SJC: swollen joint count; TJC: tender joint count; TNF: tumor necrosis factor.
} 
a mean of 4.0 DMARD (biologic and nonbiologic, including MTX) and 1.5 TNF inhibitors.

A total of 176 patients received open-label RTX $(2 \times 500$ $\mathrm{mg}$ ) in combination with a biologic DMARD. Of those, 160 (90.9\%) completed 24 weeks and $134(76.1 \%)$ completed 48 weeks of the study. The most common reasons for withdrawal were the patient's own decision, followed by AE (Table 2).

Patients received RTX with biologic and nonbiologic DMARD in 18 different combinations (Table 3). In addition to RTX, 110 patients $(62.5 \%)$ received biologics alone while 66 patients $(37.5 \%)$ received biologics with nonbiologic DMARD. The most common combinations were with only adalimumab $(n=46 ; 26.1 \%)$ and only etanercept $(n=37$; $21.0 \%$ ). The assessment population included 11 patients who had deviated from the protocol. Ten of those patients received RTX in combination with infliximab monotherapy,

Table 2. Patient disposition up to Week 48 (safety population).

\begin{tabular}{lc}
\hline & $\begin{array}{c}\text { No. Patients }(\%), \\
\text { Total }=176\end{array}$ \\
\hline Completed Week 48 & $134(76.1)$ \\
Discontinued before/at Week 48 & $42(23.9)$ \\
Reason for withdrawal & \\
$\quad$ Patient's decision* & $16(9.1)$ \\
Adverse event & $13(7.4)$ \\
Physician's decision & $6(3.4)$ \\
Lost to followup & $3(1.7)$ \\
Pregnancy & $1(0.6)$ \\
Death & $3(1.7)$ \\
\hline
\end{tabular}

* Patient withdrew for reasons other than adverse event.

Table 3. Biologic and nonbiologic disease-modifying antirheumatic drugs used in combination with rituximab.

\begin{tabular}{lc}
\hline Agent(s) & $\begin{array}{c}\text { No. Patients }(\%), \\
\text { Total }=176\end{array}$ \\
\hline Adalimumab & $46(26.1)$ \\
Adalimumab + MTX & $16(9.1)$ \\
Adalimumab + leflunomide & $2(1.1)$ \\
Adalimumab + infliximab* & $1(0.6)$ \\
Adalimumab + MTX + HCQ & $2(1.1)$ \\
Etanercept & $37(21.0)$ \\
Etanercept + MTX & $21(11.9)$ \\
Etanercept + leflunomide & $2(1.1)$ \\
Etanercept + HCQ & $1(0.6)$ \\
Etanercept + sulfasalazine & $1(0.6)$ \\
Etanercept + MTX + HCQ & $2(1.1)$ \\
Etanercept + MTX + sulfasalazine & $1(0.6)$ \\
Abatacept & $17(9.7)$ \\
Abatacept + MTX & $7(4.0)$ \\
Abatacept + MTX + HCQ & $2(1.1)$ \\
Infliximab* & $10(5.7)$ \\
Infliximab + MTX & $7(4.0)$ \\
Infliximab + leflunomide & $1(0.6)$ \\
\hline
\end{tabular}

* protocol deviation. MTX: methotrexate; HCQ: hydroxychloroquine. and 1 patient received RTX with a combination of adalimumab and infliximab (Table 3).

Safety. There were 19 SAE observed in 16 patients (9.1\%) during the first 24 weeks of the study, corresponding to a rate of 24.3 events per 100 patient-years (95\% CI 15.5-38.1). There were no trends in the types of SAE reported, either overall or with regard to any particular biologic treatment combination with RTX. With abatacept, there were $5 \mathrm{SAE}$ (3 with abatacept alone and 2 with abatacept, MTX, and hydroxychloroquine). Six SAE were reported for combination with adalimumab (5 with adalimumab alone and 1 with adalimumab and MTX). There were 4 SAE for RTX in combination with etanercept alone, and a further $4 \mathrm{SAE}$ for the combination with infliximab and MTX.

Of the 19 SAE that occurred during the first 24 weeks of the study, 3 were classified as SIE and occurred in 3 patients [rate of 3.8 events per 100 patient-yrs (95\% CI 1.2-11.9)]. Cellulitis occurred twice (the first time in the left arm and the second time, 6 weeks later, in the right leg) in 1 patient who received a single RTX infusion in addition to abatacept, MTX, and hydroxychloroquine; this patient withdrew from treatment 11 days later and entered safety followup. Pneumonia occurred in a second patient who had received abatacept plus 2 courses of RTX; this event was considered resolved 4 days later and no subsequent action was taken with regard to the study drug. The third patient experienced bronchitis that resolved after 1 day; the patient had received 2 courses of RTX plus adalimumab.

Between Weeks 24 and 40, 147 patients (83.5\%) were retreated with RTX. In this subset of patients, 10 SAE were reported in 9 patients $(6.1 \%)$, which corresponds to a rate of 16.2 (95\% CI 8.7-30.1) events per 100 patient-years. The overall SIE rate at 48 weeks was 2.7 events per 100 patient-years (95\% CI 1.0-7.2), with 1 SIE occurring between 24 and 48 weeks (a total of 4 SIE overall) in a patient who had been receiving infliximab plus 2 courses of RTX. This patient was recorded as having septic shock that resolved after 10 days.

Three deaths occurred within the 48-week study period. One patient, who had received RTX plus etanercept, died 6 months after last dose of the study drug following a ruptured aortic aneurysm. The second death occurred in a 66-year-old patient with hypertension and renal insufficiency, who had received RTX plus abatacept and MTX. This patient was hospitalized for treatment of deep venous thrombosis 7 months after the last dose of study drug, and was reported to have died as a result of respiratory failure during the hospitalization. The cause of the respiratory failure was not reported. The third death occurred in a 78-year-old patient with multiple comorbidities who had received RTX plus adalimumab. This patient was admitted to the hospital with dehydration and hyponatremia, possibly secondary to emesis, 3 weeks after her last known dose of the study drug.

Personal non-commercial use only. The Journal of Rheumatology Copyright @ $\subsetneq$ 2013. All rights reserved. 
Death due to cardiopulmonary arrest occurred about 2 weeks later; details of this event are limited. None of the 3 deaths was reported to be due to infections. A further 3 deaths were reported in the database after completion of the 48-week treatment period. One patient who had received RTX with concomitant MTX and etanercept died from lung cancer 2 years after last dose of the study drug. Another patient died about 20 months after receiving last dose of the study drug with concomitant infliximab (primary cause of death not reported). The third death occurred in a patient who had received concomitant adalimumab; cause of death was recorded as pneumonia and occurred about 21 months after last dose of study drug.

The frequency of IRR was lower with the second course of RTX compared with the first, and lower with the second infusion of RTX than with the first infusion within each course $(31.3 \%$ and $16.5 \%$ in course $1 ; 15.6 \%$ and $10.9 \%$ in course 2; Table 4). No serious IRR were reported.

A total of 14 patients $(8.0 \%)$ tested positive for HACA during the 48 -week treatment period. After testing HACA-positive, 4 patients experienced a total of 4 IRR, none of which affected the infusions or was serious. There was no clear association of HACA positivity with specific concomitant biologic DMARD. Three patients were HACA-positive at baseline, and all subsequently tested HACA-negative at 24 weeks. None of these patients experienced IRR.

Efficacy. At 24 weeks following a single course of RTX, $30.7 \%, 10.2 \%$, and $5.1 \%$ of patients achieved ACR20, ACR50, and ACR70 responses, respectively (Figure 1). ACR response rates were improved numerically at Week 48, increasing to $48.9 \%, 22.7 \%$, and $9.1 \%$, respectively. A similar trend was observed for DAS28 LDA, DAS28 remission, and EULAR responses (Figure 1). Physical function was improved compared with baseline, as shown by the mean decrease in HAQ-DI score of 0.28 at 24 weeks and 0.32 at 48 weeks.

\section{DISCUSSION}

These results from the SUNDIAL II study indicate that the overall safety profile of RTX $2 \times 500 \mathrm{mg}$ when used in combination with a single biologic DMARD over 48 weeks is consistent with that reported previously in studies of RTX plus MTX or other nonbiologic DMARD ${ }^{10,14,16}$ and in a study of RTX plus biologic DMARD ${ }^{15}$. The safety profile was consistent at 24 and 48 weeks, with the majority of patients receiving a second course of RTX during the study. The choice of $500 \mathrm{mg} \times 2$ instead of $1000 \mathrm{mg} \times 2$ was based on the concern that previous studies using 2 biologics in the treatment of RA had shown increased rates of infection, even at doses lower than standard for both agents ${ }^{8,9}$. In the current study, patients continued the standard doses of their biologic that they had been taking prior to addition of RTX. Thus, it is striking that no evidence of an increased safety signal was observed. Subsequent studies in MTX-refractory and MTX-naive populations have established that the safety profiles of RTX at 500 and $1000 \mathrm{mg}$ dosing schedules are similar $^{13,17}$.

This safety profile should be considered in the context of this treatment-refractory patient population, as evidenced by the relatively long disease duration (mean about 11 years) and previous treatment with an average of $1.5 \mathrm{TNF}$ inhibitors. Interestingly, the rates of SAE and SIE reported were consistent with those of previous studies in similar

Table 4. Summary of safety over 48 weeks after treatment with biologic and nonbiologic disease-modifying antirheumatic drugs in combination with rituximab.

\begin{tabular}{lccc}
\hline Safety Population & $\begin{array}{c}\text { No. Patients }(\%) \\
\text { Total }=176\end{array}$ & Events & $\begin{array}{c}\text { Rate/100 Patient-yrs (95\% CI) } \\
\text { Total Patient-yrs = 147.2 }\end{array}$ \\
\hline AE & $164(93.2)$ & 921 & $625.7(586.6-667.4)$ \\
SAE & $27(15.3)$ & 33 & $22.4(15.9-31.5)$ \\
IRR* & $55(31.3)$ & 103 & - \\
$\quad$ First infusion & $29(16.5)$ & 50 & - \\
$\quad$ Second infusion & $104(59.1)$ & 217 & $147.4(129.1-168.4)$ \\
Infections & $4(2.3)$ & 4 & $2.7(1.0-7.2)$ \\
Serious infections ${ }^{\dagger}$ & $\mathrm{n}=147$ & & Total Patient-yrs $=61.7$ \\
& $105(71.4)$ & 312 & $505.8(452.7-565.1)$ \\
Retreatment Population & $9(6.1)$ & 10 & $16.2(8.7-30.1)$ \\
AE & $23(15.6)$ & 40 & - \\
SAE & $16(10.9)$ & 30 & - \\
IRR & $49(33.3)$ & 72 & $116.7(92.7-147.1)$ \\
$\quad$ First infusion & $1(0.7)$ & 1 & $1.6(0.2-11.5)$ \\
$\quad$ Second infusion & Infections & & \\
Serious infections ${ }^{\dagger}$ & & & \\
\hline
\end{tabular}

$* \mathrm{AE}$ that occurred within $24 \mathrm{~h}$ of rituximab infusion. ${ }^{\dagger} \mathrm{SAE}$ or any infection that was treated with an intravenous antibiotic. AE: adverse event; SAE: serious AE; IRR: infusion-related reaction. 


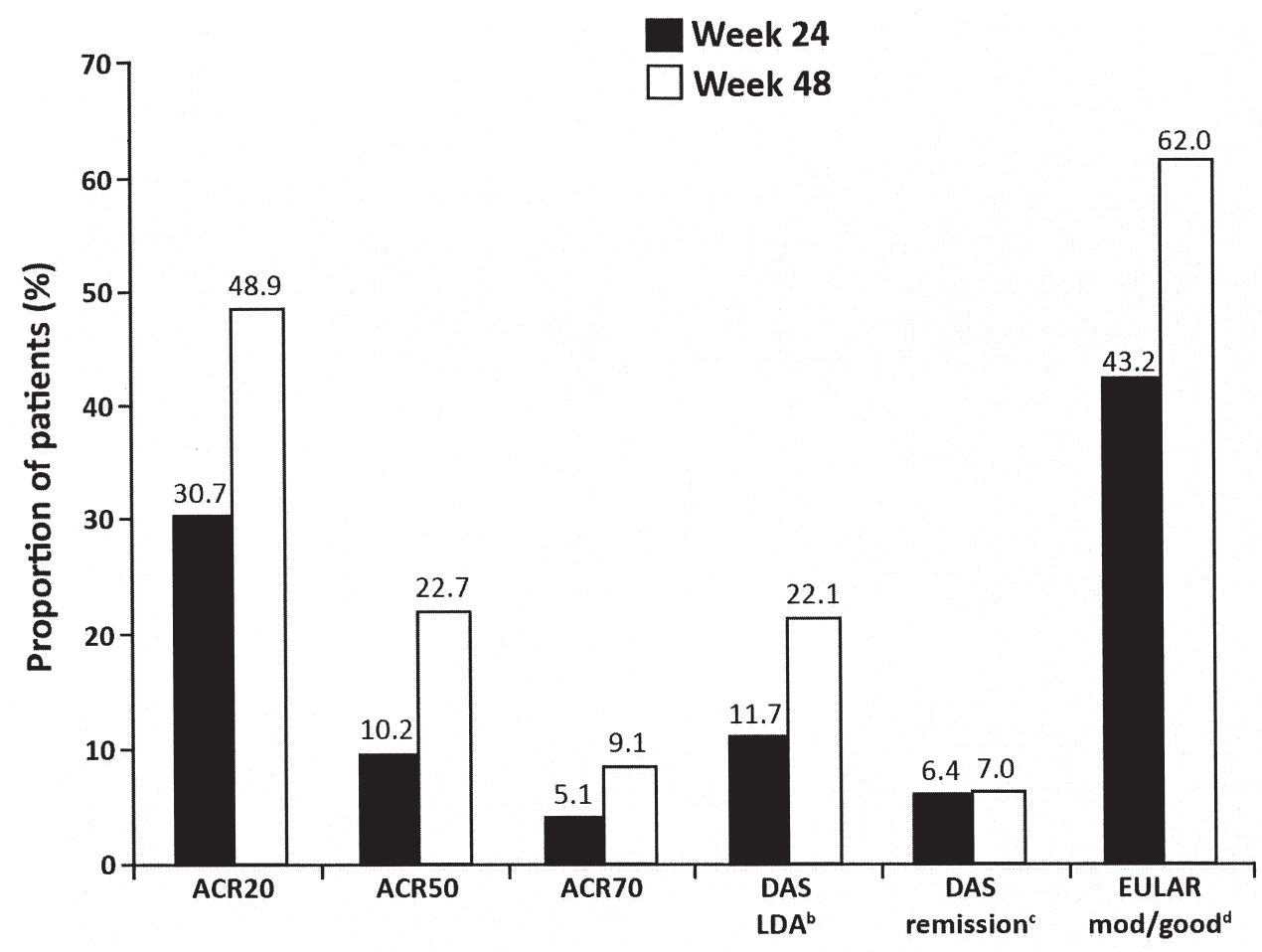

Figure 1. Efficacy in patients who received biologic and nonbiologic disease-modifying antirheumatic drugs in combination with rituximab. ${ }^{a}$ Last-observation carried-forward method was used to impute post-baseline missing ACR components. Subjects with insufficient data to calculate ACR20/ACR50/ACR70 responses or who withdrew from the study prior to assessment were classified as nonresponders. ${ }^{b}$ DAS-ESR $\leq 3.2 .{ }^{c}$ DAS-ESR $<$ 2.6. ${ }^{\mathrm{d} E U L A R}$ moderate and good responses were achieved by $34.1 \%$ and $9.1 \%$ of patients at Week 24 , and $43.8 \%$ and $18.2 \%$ of patients at Week 48, respectively. ACR: American College of Rheumatology; DAS: Disease Activity Score; ESR: erythrocyte sedimentation rate; EULAR: European League Against Rheumatism; LDA: low disease activity.

patient populations in which RTX was used as the sole biologic agent. In the current study, the 24-week rates of SAE (24.3 events/100 patient-yrs) and SIE (3.8 events/100 patient-yrs) were similar to those seen following 24 weeks of RTX treatment in the REFLEX study (21.6 and 5.2 events/100 patient-yrs, respectively), which involved patients refractory to TNF inhibitors and with similarly long disease duration (about $12 \mathrm{yrs})^{10}$. Similarly, the incidence of SAE $(6.1 \%)$ and SIE $(0.7 \%)$ in the retreatment population in the current study was consistent with those reported in the SUNRISE study ( $7 \%$ and $2 \%$, respectively), in which patients received 2 courses of RTX ${ }^{14}$. It should be noted that patients in REFLEX and SUNRISE received the approved RTX dose of $2 \times 1000 \mathrm{mg}$, in contrast to the lower $2 \times 500$ mg dose used in the current study, which is not an approved dosing regimen. Finally, the frequency of HACA with RTX in combination with another biologic DMARD was $8 \%$ in the current study, consistent with a previous pooled analysis that reported an incidence of $11 \%^{18}$. Consistent with previous reports, there was no apparent association between HACA positivity and the occurrence of IRR ${ }^{10,14}$.

Although the data suggest a potential clinical improvement with RTX in combination with another biologic
DMARD, interpretation of the efficacy results is quite limited because the current study had no placebo-control arm, and the dosing regimen, entry criteria, and baseline prognostic factors of SUNDIAL were distinct from previous RTX clinical trials conducted in patients who did not respond to TNF inhibitors (REFLEX ${ }^{10}$ and SUNRISE ${ }^{14}$ ). The safety profile of RTX $2 \times 500 \mathrm{mg}$ in combination with 1 other biologic DMARD was consistent with that reported previously for RTX plus MTX or other nonbiologic DMARD $^{10,14,16}$.

\section{ACKNOWLEDGMENT}

We thank all the SUNDIAL investigators.

\section{REFERENCES}

1. van Vollenhoven RF. Treatment of rheumatoid arthritis: State of the art 2009. Nat Rev Rheumatol 2009;5:531-41.

2. Weinblatt ME, Kremer JM, Bankhurst AD, Bulpitt KJ, Fleischmann RM, Fox RI, et al. A trial of etanercept, a recombinant tumor necrosis factor receptor: $\mathrm{Fc}$ fusion protein, in patients with rheumatoid arthritis receiving methotrexate. N Engl J Med 1999;340:253-9.

3. Lipsky PE, van der Heijde DM, St. Clair EW, Furst DE, Breedveld FC, Kalden JR, et al, and the Anti-Tumor Necrosis Factor Trial in Rheumatoid Arthritis with Concomitant Therapy Study Group.

$$
\text { Personal non-commercial use only. The Journal of Rheumatology Copyright } \subset \text { C 2013. All rights reserved. }
$$


Infliximab and methotrexate in the treatment of rheumatoid arthritis. N Engl J Med 2000;343:1594-602.

4. Weinblatt ME, Keystone EC, Furst DE, Moreland LW, Weisman $\mathrm{MH}$, Birbara CA, et al. Adalimumab, a fully human anti-tumor necrosis factor monoclonal antibody, for the treatment of rheumatoid arthritis in patients taking concomitant methotrexate: The ARMADA trial. Arthritis Rheum 2003;48:35-45.

5. Keystone EC, Schiff MH, Kremer JM, Kafka S, Lovy M, DeVries $\mathrm{T}$, et al. Once-weekly administration of $50 \mathrm{mg}$ etanercept in patients with active rheumatoid arthritis: Results of a multicenter, randomized, double-blind, placebo-controlled trial. Arthritis Rheum 2004;50:353-63.

6. Schiff M, Keiserman M, Codding C, Songcharoen S, Berman A, Nayiager S, et al. Efficacy and safety of abatacept or infliximab vs placebo in ATTEST: A phase III, multi-centre, randomised, double-blind, placebo-controlled study in patients with rheumatoid arthritis and an inadequate response to methotrexate. Ann Rheum Dis 2008;67:1096-103.

7. Mewar D, Wilson AG. Treatment of rheumatoid arthritis with tumour necrosis factor inhibitors. Br J Pharmacol 2011;162:785-91.

8. Genovese MC, Cohen S, Moreland L, Lium D, Robbins S, Newmark R, et al, for the 20000223 Study Group. Combination therapy with etanercept and anakinra in the treatment of patients with rheumatoid arthritis who have been treated unsuccessfully with methotrexate. Arthritis Rheum 2004;50:1412-9.

9. Weinblatt M, Combe B, Covucci A, Aranda R, Becker JC, Keystone E. Safety of the selective costimulation modulator abatacept in rheumatoid arthritis patients receiving background biologic and nonbiologic disease-modifying antirheumatic drugs: A one-year randomized, placebo-controlled study. Arthritis Rheum 2006;54:2807-16.

10. Cohen S, Emery P, Greenwald M, Dougados M, Furie R, Genovese $\mathrm{M}$, et al, for the REFLEX trial group. RTX for rheumatoid arthritis refractory to anti-tumor necrosis factor therapy: Results of a multicenter, randomized, double-blind, placebo-controlled, phase III trial evaluating primary efficacy and safety at twenty-four weeks. Arthritis Rheum 2006;54:2793-806.

11. Cohen SB, Keystone E, Genovese MC, Emery P, Peterfy C, Tak PP, et al. Continued inhibition of structural damage over 2 years in patients with rheumatoid arthritis treated with rituximab in combination with methotrexate. Ann Rheum Dis 2010;69:1158-61.
12. Emery P, Fleischmann RM, Filipowicz-Sosnowska A, Szczepanski L, Kavenaugh A, Racewicz AJ, et al. The efficacy and safety of rituximab in patients with active rheumatoid arthritis despite methotrexate treatment: Results of a phase IIb double-blind, placebo-controlled, dose-ranging trial (DANCER). Arthritis Rheum 2006;54:1390-400.

13. Emery P, Deodhar A, Rigby WF, Isaacs JD, Combe B, Racewicz $\mathrm{AJ}$, et al. Efficacy and safety of different doses and retreatment of rituximab: A randomised, placebo-controlled trial in patients who are biological naive with active rheumatoid arthritis and an inadequate response to methotrexate (Study Evaluating Rituximab's Efficacy in MTX iNadequate rEsponders (SERENE)). Ann Rheum Dis 2010;69:1629-35.

14. Mease PJ, Cohen S, Gaylis NB, Chubick A, Kaell AT, Greenwald $\mathrm{M}$, et al. Efficacy and safety of retreatment in patients with rheumatoid arthritis with previous inadequate response to tumor necrosis factor inhibitors: Results from the SUNRISE trial. J Rheumatol 2010;37:917-27.

15. Greenwald MW, Shergy WJ, Kaine JL, Sweetser MT, Gilder K, Linnik MD. Evaluation of the safety of rituximab in combination with a tumor necrosis factor inhibitor and methotrexate in patients with active rheumatoid arthritis: Results from a randomized controlled trial. Arthritis Rheum 2011;63:622-32.

16. Loveless J, Olech E, Pritchard CH, Chai A, Kelman A, Klearman $\mathrm{M}$, et al. An open-label, prospective study (SUNDIAL) of the safety of rituximab in combination with disease-modifying anti-rheumatic drugs in patients with active rheumatoid arthritis (SUNDIAL) [abstract]. Arthritis Rheum 2009;60 Suppl:S1660.

17. Tak PP, Rigby WF, Rubbert-Roth A, Peterfy CG, van Vollenhoven $\mathrm{RF}$, Stohl W, et al; IMAGE Investigators. Inhibition of joint damage and improved clinical outcomes with rituximab plus methotrexate in early active rheumatoid arthritis: The IMAGE trial. Ann Rheum Dis 2011;70:39-46.

18. van Vollenhoven RF, Emery P, Bingham CO III, Keystone EC, Fleischmann R, Furst DE, et al. Longterm safety of patients receiving rituximab in rheumatoid arthritis clinical trials. J Rheumatol 2010;37:558-67. 